\title{
Editorial
}

J.W.R. McIntyre MD FRCPC

\section{Tracheal intubation and laryngoscope design}

In this issue of the Journal, Marks et al. report measured characteristics of some laryngoscope blades. Their study should be discussed within the context of tracheal intubation because of its implications for that process. The measurements supplement the steadily increasing knowledge of anatomy, anaesthesia techniques, problem anticipation, and helpful devices that has accumulated during the past decade.

The functional anatomy of the larynx is well documented, ${ }^{1-3}$ as is its relationship with the cervical spine. ${ }^{4}$ Though not formally described, anaesthetists know that external manipulation of the larynx changes the relative position of certain anatomical structures and that may improve visualisation and access to the vocal cords. The preanaesthetic history and examination can almost certainly indicate problems that will be encountered when intubation is attempted. It is in this anticipation of intubation problems that great advances have been made. Imaging ${ }^{5}$ and a variety of ingenious devices ${ }^{6-8}$ are largely inappropriate for routine use and may not identify every problem. Careful clinical examination is vital. Adverse signs classically described have now been defined more accurately and supplemented by others whose predictive value have been established by clinical studies.

Consideration of that clinical information enables the anaesthetist to identify the need for fibreoptic or other special techniques. Identifying the laryngoscope for other patients is more difficult. The best choice enables the anaesthetist to obtain the optimum possible view and access to the vocal cords without stressing teeth or temporamandibular joints. ${ }^{9}$ Marks et al. have described a laboratory method, based on $x$-rays of normal patients, that measures two characteristics of certain laryngoscope blades: the amount of space that the blade occupies behind the mandible and the deviation of the line of vision from the ideal at laryngoscopy. These are important when trying to intubate the "anterior larynx." Their findings have helped explain why sometimes a distal portion of a large and curved blade is more effective than the full

From the University of Alberta, Edmonton. length of a shorter one. It should be noted that the study utilised Macintosh (Penlon) blades. All Macintosh blades have a parabolic curve but there is some variation among commercially available products and thus, presumably, differences among them in measurements and derived calculations. It remains to be elucidated whether the anatomical characteristics in the subjects studied are similar in all races. Certainly the flatter Asiatic faces can contribute to intubation problems by denying the opportunity to shorten the distance, and alter the angle of approach to the vocal cords by fully utilising the corner of the mouth.

The authors tell us that, lacking certain measurements requiring radiography, it is impossible to state what the undesirable values are in a particular clinical circumstance. Their difficulty is aggravated because the measurements upon which the calculations are based might be altered by the cricoid pressure applied routinely to many patients. The position of point $\mathrm{T}$ would possibly alter. One of the dilemmas of anaesthetists is the adverse effects of cricoid pressure applied in Sellick's neck position, on visualization and access to the vocal cords. Additionally point $S$ will be different in the presence of underbite which is a fairly common finding in some populations.

The apparently limited value of the measurements MIT and EIT in the published study and the absence of attention to details of the blade step and flange may tempt busy anaesthetists to dismiss the study as clinically irrelevant, particularly if their clinical experience leads them to reject the authors' claim that a curved blade is better than a straight one. This would do the authors an injustice. In spite of a vast helpful literature, attempted tracheal intubation remains a source of critical incident, trauma, and mortality. This could be reduced by descriptive studies incorporating details of anticipated problems, measurements of teeth pressure ${ }^{10}$ and temporamandibular joint strain, ${ }^{11}$ and outcome, as well as functional measurements of the laryngoscope blade used. Such a study in a solitary institution may not supply statistically significant findings regarding a certain blade but by em- 
ploying criteria based on current knowledge it is an indication of the quality of care available in that institution. The variation in clinical practice that has been identified in other studies ${ }^{12,13}$ may be because there is more than one ideal way of doing the same thing or due to a variation in skills that influences the incidence of critical incidents, trauma, and mortality. What is "difficult" to one anaesthetist may be "easy" to another. It is a subjective term meaning "hard to do" or "troublesome." It alludes to the skill of the anaesthetist as much as the patient's anatomy, and existence of the term "difficult intubation" implies that, inevitably, the occasional struggle is to be expected. Perhaps the term "problem intubation" should be adopted. "Problem" suggests that the process presents unusual characteristics to be taken into consideration and which can be solved by persons possessing the necessary knowledge and skills.

Serious challenges face those teaching tracheal intubation to novices and refreshing the skills of practitioners. There is a substantial body of information to impart and at the termination of training the trainees should be able to identify the clinical management in which they are competent and what they have not yet learned to do. Ethical considerations are demanding and clinical experience is severely restricted by the need to protect patient and anaesthetist from the activities of trainees. Fortunately, much can be learned outside the operating room and without touching patients. A plan for tracheal intubation depends on matching objectives, educational methods, and progress evaluation. ${ }^{14-16}$ It is likely to have better long-term results than can be produced by a relatively unstructured approach confined to the operating room.

In conclusion the functional analyses of some laryngoscope blades reported by Marks et al. begins a rational analysis of function that will encourage anaesthetists to select the most suitable blade for their purpose, not merely a blade with which they can just manage successfully. In a training milieu its value lies in the period before experience is gained with patients and should further reduce the morbidity and mortality presently associated with tracheal intubation.

\section{Les lames de laryngoscope et leur conception}

Dans un article qui paraît dans ce numéro du Journal, Marks et se collègues se sont appliqués à mesurer les dimensions de quelques modèles de lames de laryngoscope. Pour des raisons évidentes, c'est dans le contexte de l'intubation endotrachéale qu'il faut discuter cette étude. Ce travail vient s'ajouter à la masse des connaissances acquises pendant la dernière décennie sur l'anatomie, les techniques anesthésiques, l'anticipation des problèmes et le perfectionnement du matériel.

L'anatomie functionnelle du larynx est bien documentée $e^{-3}$ ainsi que ses rapports avec la colonne cervicale. ${ }^{4}$ Les anesthésistes connaissent bien les effets sur le larynx des manipulations externes qui changent la position relative des structures anatomiques. Ils peuvent ainsi améliorer la visualisation et obtenir une meilleur accès aux cordes vocales. L'histoire et l'examen préanesthésiques peuvent, presque à coup sûr, déceler les difficultés susceptibles de survenir à l'intubation. C'est vers l'anticipation de ces problèmes que des importants progrès ont été réalisés. Par ailleurs, limagerie ${ }^{5}$ et d'autres dispositifs ingénieux ${ }^{6-8}$ ne se prêtent pas à l'usage courant et ne peuvent identifier tous les problèmes. Il faut donc toujours procéder à un examen clinique minutieux: les indices défavorables classiques sont alors identifiés et on peut recourir à d'autres examens dont la valeur prévisionnelle est déjà reconnue.

Gràce à cette information, l'anesthésiste peut prévoir sil aura besoin de la fibroscopie ou d'une autre technique spéciale. Chez d'autres patients, le choix du laryngoscope est plus difficule. Le meilleur choix est celui qui permet à l'anesthésiste une vision optimale et un accès au larynx sans exercice de contrainte sur les dents ou l'articulation temporomandibulaire. ${ }^{9}$ Marks et coll. décrivent une méthode expérimentale, basée sur les radiographies de sujets normaux, qui mesure deux caractéristiques de lames de laryngoscope: l'espace qu'occupe la lame derrière le maxillaire inférieur et la déviation de la ligne de vision de l'axe idéal pendant la laryngoscopie. Ces données sont importantes pour l'intubation du larynx antérieur. Elles permettent d'expliquer que la portion distale d'une grande lame courbe est souvent plus efficace que toute la longueur d'une lame plus courte. Il faut noter que dans cette étude, on utilise des lames Macintosh de marque Penlon. Toutes les lames Macintosh ont une courbe pa- 
rabolique mais il existe des différences entre les marques disponibles et, on peut le présumer, entre les mesures et les calculs qui en découlent. Il reste aussi à déterminer si les caractéristiques anatomiques des sujets étudiés sont identiques pour toutes les races. Le facies asiatique est certainement plus aplati, ce peut contribuer aux difficultés en éloignant les cordes vocales et en altérant l'angle d'approche par le coin de la bouche.

Comme il leur manque certaines mesures acquises par radiologie, il est impossible, selon les auteurs, de déclarer quelles sont les valeurs inapplicables dans des circonstances cliniques particulières. Leur problème se complique du fait que les mesures sur lesquelles ils se basent sont modifiées par la pression circoide: vraisemblablement, dans ce cas, la position du point $\mathrm{T}$ varierait. L'anesthésiste doit aussi faire face au dilemme causé par l'effet nuisible sur la visualisation et l'accès au larynx, de la pression cricoïde exercée sur un cou en position de Sellick. De plus, la rétrognathie mandibulaire, assez fréquemment rencontrée, déplace le point $S$.

La valeur limitée apparente des mesures MIT et EIT dans l'étude et le manque d'attention aux détails peut porter l'anesthésiste à ne pas tenir compte de cette étude à cause de son manque de portée clinique; particulièrement si son expérience lui fait rejeter l'affirmation des auteurs selon laquelle une lame courbe est supérieure à une droite. Ce serait injuste envers les auteurs. Les tentatives d'intubation demeurent causes d'incidents, de traumatismes et de mortalités. La fréquence de ces accidents diminuerait si on disposait d'études descriptives incorporant des détails sur les problèmes anticipés, les mesures de la pression appliquée sur les dents, ${ }^{10}$ de la contrainte subie; par l'articulation temporomandibulaire, ${ }^{11}$ et leurs résultats, tout en faisant le rapprochement qui simpose avec la lame de laryngoscope utilisée. Une telle étude menée dans une seule institution peut ne pas fournir de données statistiquement significatives sur une lame en particulier mais en utilisant des critères basés sur nos connaissances actuelles, elle devient un indice de la qualité de l'acte. La différence de pratique identifiée dans d'autres études ${ }^{12,13}$ peut s'expliquer par le fait qu'il ny a pas qu'une façon idéale de faire une même chose; thabilité individuelle varie et influence la fréquence des incidents graves, des traumatismes et des décès. Ce qui est difficile pour un peut être facile pour l'autre. Le terme " difficile " est fortement subjectif: il signifie " exigeant ", " pénible ". Il se rapporte aussi bien à l'habileté de l'anesthésiste qu'à l'anatomie de son patient. L'expression " intubation difficile " laisse supposer que des efforts inhabituels devront être faits. Peut-être faudrait-il accepter l'expression " intubation-problèmes ».* Le term problème suggère que des caractéristiques exceptionnelles doivent être prises en considération qui ne peuvent être résolues que par des personnes possédant des connaissances et habiletés de hauts niveaux.

Ceux qui enseignent l'intubation à des novices et qui mettent à jour les connaissances de praticiens établis font face à un défi de taille. La somme d'information à transmettre est considérable et à la fin de sa formation, le résident devrait reconnaître ses limites. L'éthique est exigeante, et l'acquisition de l'expérience clinique doit être restreint par la nécessité de protéger le patient et le patron des essais intempestifs d'un apprenti. Heureusement, on peut apprendre beaucoup en dehors de la salle d'opération sans avoir de contacts avec un patient. Un plan de formation pour l'intubation trachéale nécessite des objectifs et des méthodes pédagogiques appropriés, et une évaluation dynamique. ${ }^{14-16}$ On peut s'attendre ainsi à obtenir de meilleurs résultats à long terme que ceux qui peuvent être acquis par une approche mal structurée et confinée à la salle d'opération.

Pour conclure, l'analyse fonctionnelle de quelques lames de laryngoscope rapportée par Marks et coll. est un début qui encouragera l'anesthésiste à choisir la lame la mieux adaptée à ses besoins, au lieu de celle avec laquelle il connait ordinairement du succès. Pour le milieu d'enseignement, son application se situe avant la période d'acquisition de l'expérience sur des patients et elle devrait ainsi réduire la morbidité et la mortalité attachées à l'intubation.

*Note du traducteur: l'auteur de l'éditorial aborde dans les lignes précédentes une question de sémantique qui concerne la terminoligie anglaise (difficult intubation vs problem intubation). L'adject " difficile " n'est pas plus précis que " difficult ". Cette traduction se veut fidèle mais n'a pas la prétention d'innover. Le terme « intubation-problème " n'est utilisé que pour les besoins de la cause. Aux linguistes de se prononcer.

\section{References}

1 Roberts JT. Functional anatomy of the larynx. Int Anesthesiology Clin 1990; 28: 101-5.

2 Fink BR, Demarest RJ. Laryngeal Biomechanics. Cambridge Mass: Harvard University Press. 1978.

3 Sivarajan M, Fink BR. The position and state of the larynx during general anesthesia and muscle paralysis. Anesthesiology 1990; 72: 439-42.

4 Crosby ET, Lui A. The adult cervical spine: implications for airway management. Can J Anaesth 1990; 37: 77-93.

5 Shackleford GD. The pediatric airway: radiology and new imaging. Int Anesthesiol Clin 1988; 26: 3-5.

6 Patil VU, Stehling $L C$, Zader $H L$. Predicting the difficulty of intubation using an intubation gauge. Anesthe Rev 1983; 10: $32-3$.

7 Roberts $J T$, Ali HH, Shorten GD. Using the laryngeal in- 
dices caliper to predict difficult intubations Anesthesiology 1990; 73: A1011.

8 Roberts JT, Ali HH, Shorten GD. Using the bubble inclinometer, a simple inexpensive device, to predict difficult intubations. Anesthesiology 1990; 73: A1-35.

9 Knibbe MA, Carter JP, Frockjer GM. Postanesthetic mandibular joint dysfunction. Anesth Prog 1989; 36: 21-5.

10 Racz $G B$, Allen $F B$. A new pressure sensitive laryngo. scope. Anesthesiology 1985; 62: 356-8.

11 Bishop MJ, Harrington RM, Tencer AF. Force applied during tracheal intubation. Anesth Analg 1992: 74: 411-4.

12 Horton WA, Fah Y, Charters P. Defining a standard intubating position using Anglefinder. Br J Anaesth 1989; 62: 6-12.

13 Williams KN, Carli F, Cormack RS. Unexpected, difficult laryngoscopy: a prospective survey in routine general surgery. Br J Anaesth 1991; 66: 38-44.

14 Stewart RD, Paris PM, Pelton GH. Effects of varied training techniques on field intubation success rates. Ann Emerg Med 1984; 13: 1032-6.

15 Johnston C, Roberts JT. Clinical competence in the performance of fiberoptic bronchoscopy and endotracheal intubation: a study of resident instruction. J Clin Anaesth 1989; 1: 344-9.

$16 \mathrm{Mc}$ Intyrer JWR. Learning and teaching tracheal intubation. J Clin Anaesth Pharmacol 1991; 7: 377-96. 